\title{
Competing kinematic dynamo mechanisms in rotating convection with shear
}

\author{
Michael R. E. Proctor ${ }^{1}$ and David W. Hughes ${ }^{2}$ \\ ${ }^{1}$ Department of Applied Mathematics and Theoretical Physics, \\ University of Cambridge, Cambridge CB3 0WA, U.K. \\ email: mrep@cam.ac.uk \\ ${ }^{2}$ Department of Applied Mathematics, University of Leeds, Leeds LS2 9JT, U.K. \\ email: d.w.hughes@leeds.ac.uk
}

\begin{abstract}
Following earlier work by Hughes \& Proctor (2009) on the role of velocity shear in convectively driven dynamos, we present preliminary results on the nature of dynamo action due to modified flows derived by filtration from the full convective flow. The results suggest that filtering the flow fields has surprisingly little effect on the dynamo growth rates.
\end{abstract}

Keywords. convection, instabilities, magnetic fields, MHD, Sun: activity, Sun: magnetic fields, Sun: rotation

\section{Introduction}

The principal problem in the dynamo theory for cosmical bodies such as the Sun is to explain the occurrence of magnetic fields on scales much larger than those of the underlying motions responsible for the dynamo. Such large-scale fields are often studied within the framework of mean field electrodynamics (Moffatt 1978, Krause \& Rädler 1980), but there are significant problems in applying the theory quantitatively when (as is the case in the Sun) the magnetic Reynolds numbers are large even on the smallest scales of motion (see Cattaneo \& Hughes 2009). In that circumstance, dynamo action is predominantly of small-scale type, with the magnetic fields having scales no larger than those of the flow. It is problematical to understand how such processes are compatible with the large-scale fields that are certainly observed in nature.

An appropriate geometry in which to investigate the difference between large-scale and small-scale dynamo processes is thermal convection in a rotating plane layer. This problem has been investigated in the regime of large magnetic Reynolds numbers by Cattaneo \& Hughes (2006) and Hughes \& Cattaneo (2008). They found that at moderate rotation rates the dynamos produced were always of small-scale type, with no evidence of any significant mean emf produced by the flow in the presence of a large-scale magnetic field.

However, the Sun and other bodies possess a vigorous large-scale shear flow, and it is possible to think of a number of ways in which adding a velocity shear to the convection problem may enhance the generation of the large-scale components of the field. For example, a coherent large-scale shear may change the nature of the correlations and, in so doing, enhance the $\alpha$-effect; alternatively, even if the mean emfs remain very small, then a large shear may be able to compensate for a feeble $\alpha$-effect (or a more complicated mean-field process) to make a viable two-scale dynamo. Finally, there is the very different possibility that the interactions between the large-scale shear and the (initially) smallscale convective flow lead to a large-scale velocity that can, of itself, act as a dynamo; 
this would then be effectively a small-scale (i.e. one-scale) dynamo, but on the scale of the shear flow rather than that of the convection.

This situation was investigated by Hughes \& Proctor (2009); hereinafter HP. They found (see Figure 1 below) that adding a horizontal shear to the convection problem of Cattaneo and Hughes did indeed enhance dynamo action, in particular leading to a viable dynamo even when the convecting flow without shear was not of itself a dynamo. They also demonstrated that adding the shear did nothing to improve the coherence of the emfs produced from a large-scale magnetic field by the flow. However, from inspection of the solutions, it was not possible to resolve the question as to which of the above alternative scenarios was the one responsible for dynamo action.

In this paper we try to answer this question by comparing the (kinematic) dynamo properties of the actual convective flows with those of related flows obtained by filtration in Fourier space to eliminate all small scales, or alternatively all large scales apart from the shear itself. Remarkably, we find from the preliminary results presented here that neither filtration process has very much effect on the dynamo growth rate, and so the results can be used to support both of the alternative hypotheses above. Further work is currently in progress to clarify the situation.

The plan of the paper is as follows. In the next section we set up the problem and explain the filtration process. The results are presented in $\S 3$ and the paper concludes with a discussion.

\section{Problem description}

\subsection{Governing equations}

The underlying problem is that described in HP. We consider a plane Boussinesq convective layer $(0<x, y<\lambda d, 0<z<d)$ with rotation about the vertical axis, as described in Cattaneo \& Hughes (2006). This basic model is then extended by the inclusion of a horizontal flow of the (dimensional) form

$$
U_{0}=U_{0} f(y / d) \hat{\boldsymbol{x}}, \text { where } f(y / d)=\cos \frac{2 \pi y}{\lambda d},
$$

accomplished by replacing $\boldsymbol{u}$ with $\boldsymbol{u}+\boldsymbol{U}_{0}$ in the governing equations except for the viscous term (equivalent to forcing the flow via the momentum equation, but eliminating viscous transients). For the purposes of this paper we shall restrict attention to kinematic dynamo action, so that the back-reaction of the Lorentz forces on the convection is neglected, as is appropriate for very weak fields. Then the governing non-dimensional equations for the velocity $\boldsymbol{u}$, temperature perturbation $\theta$ and magnetic field $\boldsymbol{B}$ are

$$
\begin{aligned}
\left(\partial_{t}-\sigma \nabla^{2}\right) \boldsymbol{u}+\boldsymbol{u} \cdot \nabla \boldsymbol{u}+\sigma\left(S\left(f(y) \partial_{x} \boldsymbol{u}+f^{\prime}(y) u_{y} \hat{\boldsymbol{x}}+T a^{1 / 2} \hat{\boldsymbol{z}} \times \boldsymbol{u}\right)=-\nabla p+\sigma R a \theta \hat{\boldsymbol{z}}\right. & \\
\left(\partial_{t}-\zeta \nabla^{2}\right) \boldsymbol{B}+\boldsymbol{u} \cdot \nabla \boldsymbol{B}+S f(y) \partial_{x} \boldsymbol{B} & =\boldsymbol{B} \cdot \nabla \boldsymbol{u}+S f^{\prime}(y) B_{y} \hat{\boldsymbol{x}}, \\
\left(\partial_{t}-\nabla^{2}\right) \theta+\boldsymbol{u} \cdot \nabla \theta+S f(y) \partial_{x} \theta & =\boldsymbol{u} \cdot \hat{\boldsymbol{z}} \\
\nabla \cdot \boldsymbol{B}=\nabla \cdot \boldsymbol{u} & =0 .
\end{aligned}
$$

It should be noted that although a flow with a large-scale component (i.e. with the same spatial dependence as the 'target flow' (2.1)) does indeed occur, its amplitude may differ appreciably from $U_{0}$; the hydrodynamic state that ensues depends on interactions between the shear flow and convection and, possibly, on instabilities of the shear flow itself. Importantly, the scale of variation of this shear flow is much greater than all scales 


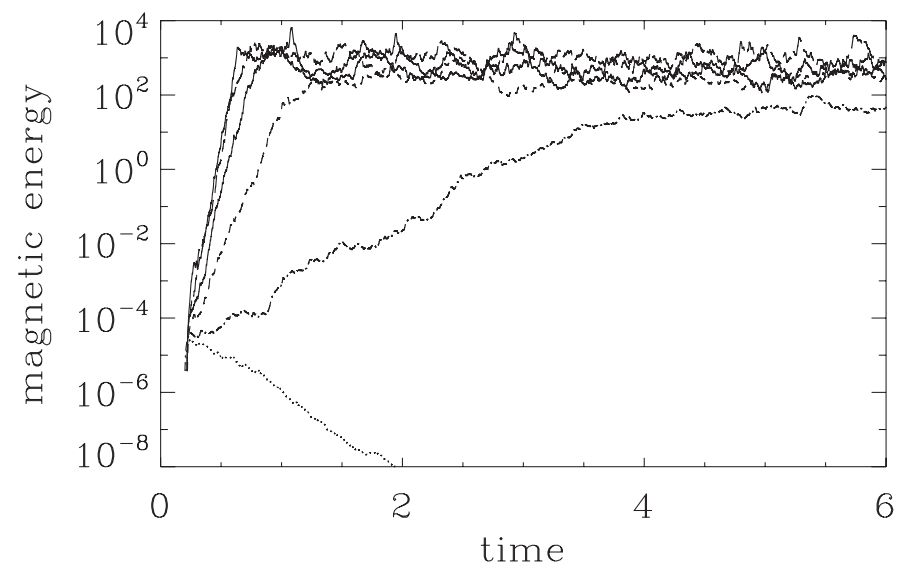

Figure 1. Evolution of magnetic energy with time for the model with the unfiltered flow and with the Lorentz force included, for various values of $S$ (from HP). The non-dynamo case is $S=1 / 3$. The case $S=5 / 3$ considered here is third from bottom.

of the convection; this is essential if the results are to be explained within the mean field framework.

The solution depends on the following dimensionless parameters: the Rayleigh number $R a=g \tilde{\alpha} \Delta T d^{3} / \kappa \nu$, proportional to the temperature difference across the layer, the Prandtl number $\sigma=\nu / \kappa$, the diffusivity ratio $\zeta=\eta / \kappa$, the Taylor number $T a=4 \Omega^{2} d^{4} / \nu^{2}$ and shear parameter $S=2 \pi U_{0} \ell / d \lambda u_{\mathrm{rms}}$, where $d$ is the layer depth, $\Delta T$ the applied temperature difference, $g$ the gravitational acceleration, $\tilde{\alpha}$ the coefficient of thermal expansion, and $\nu, \kappa, \eta$ are respectively the kinematic viscosity, thermal conductivity and magnetic diffusivity; $\ell$ and $u_{\mathrm{rms}}$ are estimates, respectively, of the horizontal scale of the convection and of the typical velocity in the absence of shear. For the particular problem below we take $S=d U_{0} / 300 \kappa$. At $z=0,1$ we take $\theta=u_{z}=\partial_{z} u_{x, y}=B_{z}=\partial_{z} B_{x, y}=0$, corresponding to fixed temperature, stress-free and perfectly conducting boundaries. We employ periodic boundary conditions in the horizontal directions.

We solve the system as an initial value problem for the convection, starting from the state of pure shear, with small random perturbations, and integrate in time until a statistically steady state is reached for the flow. We then add a weak seed magnetic field with no net flux, which will, on average, grow or decay exponentially.

In $\mathrm{HP}$ we investigated the effect of varying $S$ in the case $\lambda=5, R a=1.5 \times 10^{5}$, $T a=5 \times 10^{5}, \sigma=1, \zeta=0.2$. These parameters give vigorous convection, but there is no dynamo when $S=0$. Figure 1 shows how the dynamo growth rate increases with increasing $S$. The figure also shows the equilibration of the dynamo due to Lorentz force back reaction: this is neglected in the present paper, so that according to $(2.2)-(2.5)$, exponential growth continues indefinitely.

While it is certainly sufficient for the neglect of finite domain effects to take $\lambda=5$ when $S=0$ (see Hughes \& Cattaneo 2008), in the presence of shear some flow structures become elongated, as shown in Figure 2 below. It remains a possibility that some of the results both of HP and the present work will be modified to some degree if computations are performed in wider periodic domains. 


\subsection{Flow filtration}

Our aim in the present work is to learn more about the role of velocity shear in promoting dynamo action and the mechanism responsible for the enhancement of the growth rate. As discussed in the Introduction, the answer to such questions depends on what scales of the flow, apart from the shear, are significant in providing the interaction between the shear and the convection that powers the dynamo. Accordingly, we investigate the dynamo properties of flow fields obtained from the convective solution by a process of filtration in wavenumber space. Any Fourier mode obeying the boundary conditions has a part proportional to $\exp \pm i\left(\pi k_{z} z+2 \pi \lambda^{-1}\left(k_{x} x+k_{y} y\right)\right)$. If we denote a cut-off wave number by $k_{\text {cut }}$ then the filtration takes one of the following forms:

(a) short wavelength (SW) cutoff: set to zero the amplitudes of all modes for which $k=\max \left(\left|k_{x}\right|,\left|k_{y}\right|\right)>k_{\text {cut }}$;

(b) long wavelength (LW) cutoff: set to zero the amplitudes of all modes for which $k=\min \left(\left|k_{x}\right|,\left|k_{y}\right|\right)<k_{\text {cut }}$, but retain the mode $(0,1)$ corresponding to the shear.

To date we have performed no filtration on the vertical spectrum. This will be done in future work, though we do not consider it likely that truncating the vertical spectrum will have a significant effect.

Whatever the filtration adopted, the procedure is as follows:

(1) Solve the momentum and heat equations at full resolution;

(2) At each time step perform the filtering to produce a filtered velocity $\boldsymbol{u}_{\mathrm{f}}$ together with the shear;

(3) Solve the induction equation (2.3) at full resolution with $\boldsymbol{u}$ replaced by $\boldsymbol{u}_{\mathrm{f}}$.

\section{Results}

Our preliminary results are all for the case $S=5 / 3$, which we believe to be typical among those for which there is a dynamo. We first show the effects of filtration on the flow. Figure 2 shows a contour plot of the vertical velocity distribution near the top of the layer, with an SW cutoff and $k_{\text {cut }}=30$. The residual is shown in the right hand panel. There is little effect on the flow when $k_{\text {cut }}$ is as large as this. Figure 3 shows the effect of
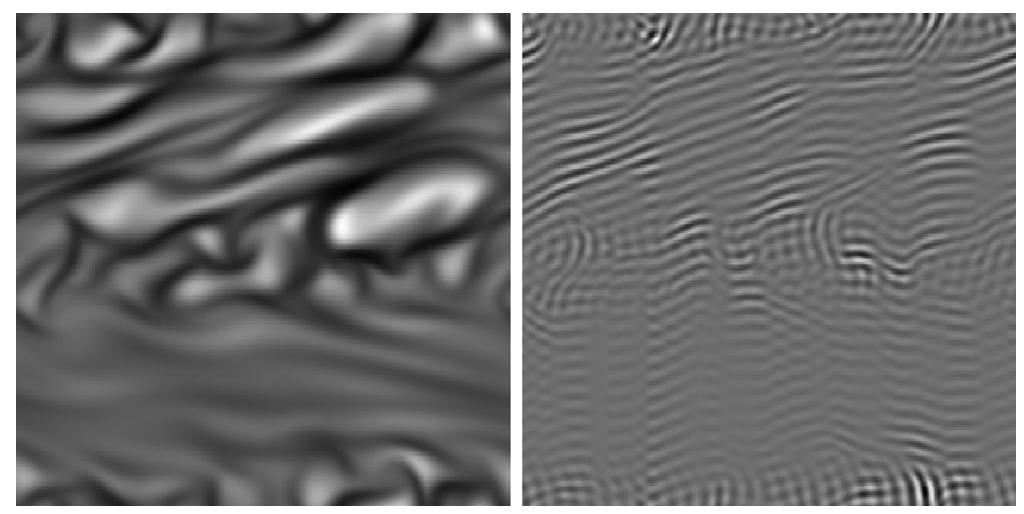

Figure 2. Left panel: Contour plot of vertical velocity near the top of the layer for a snapshot of an SW cutoff flow with $k_{\text {cut }}=30$. Right panel: the residual difference between the full and filtered flows.

changing the cutoff. By the time the cutoff wave number has been reduced to $k_{\text {cut }}=5$ the flow has been significantly altered. The (normalised) spectra of the magnetic fields 

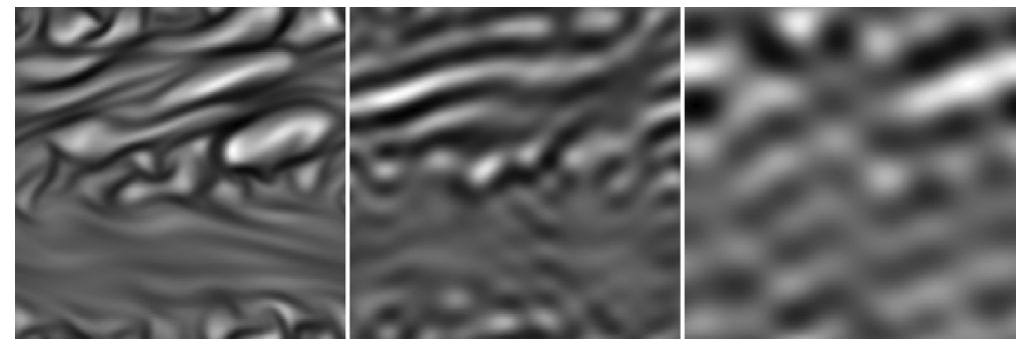

Figure 3. Effect of different SW cutoffs, for the same flow as in Figure 2. From left to right: $k_{\text {cut }}=30,10,5$. All plots are individually scaled.
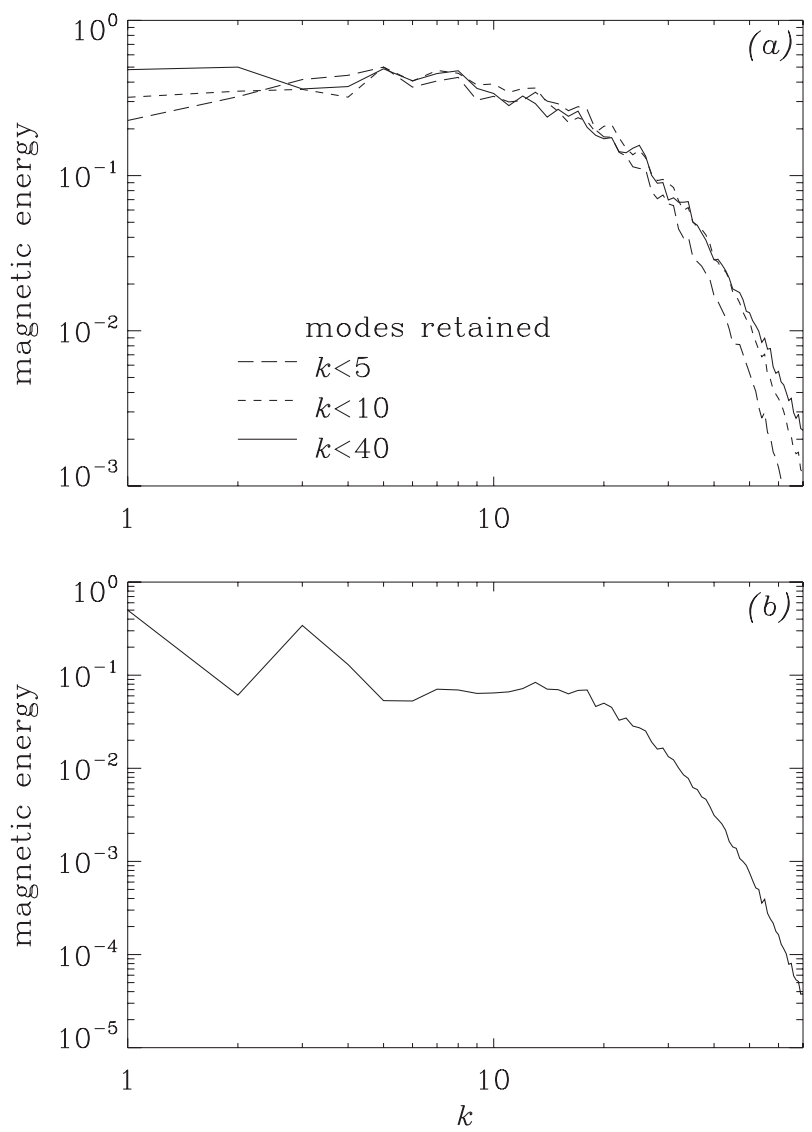

Figure 4. (a) Magnetic energy spectra for three different SW cutoffs. (b) Magnetic energy spectrum for an LW cutoff with $k_{\text {cut }}=10$.

generated by the filtered flows do not however seem to be much affected by the filtration. In Figure 4 are shown magnetic energy spectra in the interior $80 \%$ of the layer for several filtrations. For the SW cutoff (Figure $4(a)$ ) there is scarcely any effect on the energy in the modes with $5<k<20$. Figure $4(b)$ shows a similar magnetic energy spectrum for a flow with an LW cutoff. Again there seems to be little effect of the filtration except at the very largest scales.

Because of the remarkable similarities in the spectra it might then be anticipated that the growth rates for the different filtrations would not be too dissimilar. In fact they are 


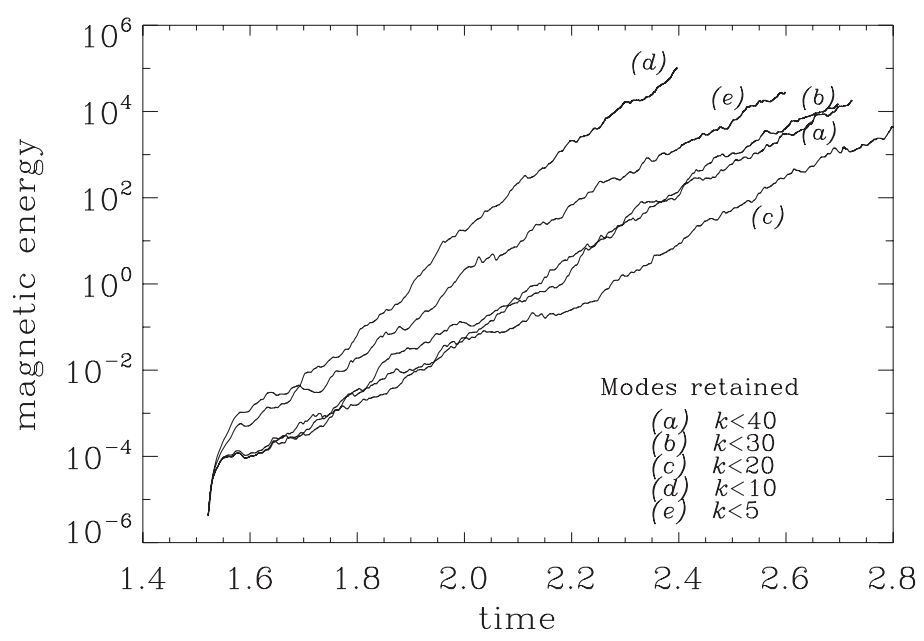

Figure 5. Time traces of magnetic energy for SW filtered flows with different values of $k_{\text {cut }}$.

hardly different at all. This is illustrated in Figures 5 and 6 , which show the growth of magnetic energy for various filtrations. The first of these compares different SW cutoffs. It would seem that removing the high wave numbers makes very little difference to the growth of the instability - though the most severe filtration does seem to lead to a greater growth rate. (The only other significant effect is that for the highly filtered flows, the system takes a little longer to settle into its ultimate exponential growth phase.) These results might lead one to speculate that the dynamo is 'small-scale on the large scale'; so that only the largest scales of motion, those comparable with the field scales, are responsible for the instability.

One's confidence in the truth of this speculation does not however survive examination of Figure 6, which shows a comparison between the growth rates for the unfiltered flow and for an LW filtration with $k_{\text {cut }}=10$. Amazingly, this flow also gives almost the same growth rate as the SW filtrations shown in Figure 5. Looking at just this figure, one might conclude that the dynamo is in fact of mean field type, since there is now a significant separation of scales in the filtered flow between the shear and what is left of the convection! Clearly more work needs to be done to distinguish between the two explanations offered above, or indeed to synthesise them.

\section{Discussion}

This paper has presented some preliminary calculations on the effects of different scales of flow on the growth rate of the dynamo instability due to rotating convection with shear. The aim of these calculations has been to ascertain just how the dynamo is being driven. On the basis of the results of HP on the unfiltered flow, we may rule out the possibility that the shear improves the coherence of the cyclonic aspects of the convection and thus produces an enhanced $\alpha$-effect. The remaining possibilities are that of a two-scale, $\alpha \Omega$ type dynamo with a strong ' $\Omega$ ' and weak ' $\alpha$ ', (or a two-scale dynamo of more complicated type) or, conversely, of a dynamo with no scale separation controlled by the flows with scales larger than the convection. Our results would appear to support either scenario!

Clearly more work has to be done to resolve the question. We certainly need to investigate larger values of the aspect ratio $\lambda$ since there is some evidence that horizontal scales of convection are approaching the current box size when shear is significant. Then more 


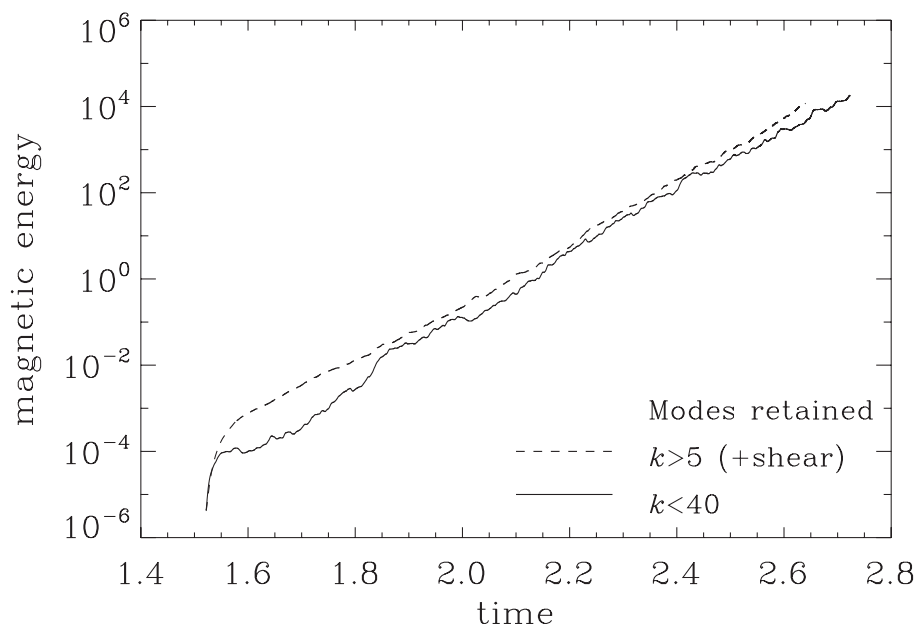

Figure 6. Increase of magnetic energy with time for (essentially) the original flow and for an LW filtration with $k_{\text {cut }}=5$.

careful filtrations at different values of $S$ are needed in order to pin down precisely which scales are really important, or whether in fact the shear controls everything and the observed growth rates are directly related to the shear rate, whatever the nature of the additional convective flow (this would of course reconcile the results shown in Figures 5 and 6 ). Investigation of the problem without rotation will lead to further insights as to whether the nature of the dynamo is two-scale (where rotation is likely to be important) or small-scale (where rotation is unimportant). It will also be of importance, particularly in view of the astrophysical implications, to repeat the calculations here and in HP for higher values of the magnetic Reynolds number, for which there is a small-scale dynamo in the absence of shear, in order to examine the role of shear in that case.

\section{Acknowledgements}

We are grateful for support from the UK STFC. The paper was prepared while the authors were invited participants in the ISIMA programme at the University of California, Santa Cruz. We are grateful to the organisers for making our attendance possible and providing an environment conducive to research.

\section{References}

Cattaneo, F. \& Hughes, D. W. 2006, J. Fluid Mech., 553, 401

Krause, F. \& Rädler, K.-H. 1980, Mean Field Magnetohydrodynamics and Dynamo Theory. New York: Pergamon Press

Hughes, D. W. \& Cattaneo, F. 2008, J. Fluid Mech., 594, 445

Hughes, D. W. \& Proctor, M. R. E. 2009, Phys. Rev. Lett., 102:044501

Moffatt, H. K. 1978, Magnetic Field Generation in Electrically Conducting Fluids. Cambridge: University Press

\section{Questions}

Steven Tobias: When filtering the equations, which scales are important? Are these scales dependent on $R m$; what is the value of $R m$ ? 
We find that paradoxically both large and small scale parts of the flow seem to be important in determining the growth rate. Rm depends on the shear rate, but for the underlying convection is of the order of 300 .

Juri Toomre: What is the role of reintroducing structures by the shear since your $x$ direction is periodic?

We have not investigated other boundary condition in $x$. Periodic boundaries are appropriate if the shear is taken to mimic zonal flows in the Sun, for example.

Geoff Vasil: How do you think that the critical shear would need to scale as a function of the Rossby number (for a fixed $R a / R a_{c}$ )?

Our feeling is that the shear should be comparable with the rotation rate for a significant change in the growth rate.

Keith Moffatt: It seems to me very likely that this is an $\alpha \Omega$ dynamo. Do your results exclude this interpretation or not?

One of our filtrations gives support to the mean field hypothesis, while the other (with all small scales removed) is just as good a dynamo! So the jury is out. 